\title{
De Lange syndrome: subjective and objective comparison of the classical and mild phenotypes
}

\author{
Judith E Allanson, Raoul C M Hennekam, Maggie Ireland
}

\begin{abstract}
Classical de Lange syndrome presents with a striking face, pronounced growth and mental retardation, and variable limb deficiencies. Over the past five years, a mild variant has been defined, with less significant psychomotor retardation, less marked pre- and postnatal growth deficiency, and an uncommon association with major malformations, although mild limb anomalies may be present. We have evaluated 43 subjects with de Lange syndrome, 30 with classical features, aged from birth to 21 years, and 13 with the mild phenotype, aged from 18 months to 30 years. In addition to assessment of gestalt and facial change with time, detailed craniofacial measurements have been obtained on each subject and composite pattern profiles compiled.

The characteristic face of classical de Lange syndrome is present at birth and changes little throughout life, although there is some lengthening of the face with age and the jaw becomes squared. In mild de Lange syndrome, the characteristic classical appearance may be present at birth, but in some subjects it may be two or three years before the typical face is obvious. In general, the overall impression is less striking, perhaps because of increased facial expression and greater alertness. With age, the face loses the characteristic appearance, the nasal height increases, the philtrum does not seem as long, and the upper vermilion is full and everted, although the crescent shaped mouth with downturned corners remains. Eyebrows may be full and bushy.
\end{abstract}

Table 1 Anthropometric measurements used in this study

\begin{tabular}{|c|c|c|}
\hline Head width & eu-eu & eurion to eurion \\
\hline Skull base width & $t-t$ & tragion to tragion \\
\hline Minimum frontal width & $\mathrm{mf}-\mathrm{mf}$ & frontotemporale to frontotemporale \\
\hline Upper facial width & $z y-z y$ & zygion to zygion \\
\hline Lower facial width & go-go & gonion to gonion \\
\hline Head length & g-op & glabella to opisthocranion \\
\hline Upper facial depth & $t-n$ & tragion to nasion \\
\hline Midfacial depth & $t-s n$ & tragion to subnasale \\
\hline Lower facial depth & $t-g n$ & tragion to gnathion \\
\hline Nasal protrusion & sn-prn & subnasale to pronasale \\
\hline Total facial height & n-gn & nasion to gnathion \\
\hline Nasal height & $\mathrm{n}-\mathrm{sn}$ & nasion to subnasale \\
\hline Nasal width & al-al & alare to alare \\
\hline Mouth width & ch-ch & cheilion to cheilion \\
\hline Inner canthal distance & en-en & endocanthion to endocanthion \\
\hline Outer canthal distance & ex-ex & exocanthion to exocanthion \\
\hline Ear width & pra-pa & preaurale to postaurale \\
\hline Ear length & sa-sba & superaurale to subaurale \\
\hline Maxillary arc & $t-s n-t$ & tragion to subnasale to tragion \\
\hline Mandibular arc & $t-g n-t$ & tragion to gnathion to tragion \\
\hline Head circumference & OFC & $\begin{array}{l}\text { Maximum circumference in horizontal plane at level } \\
\text { of glabella and opisthocranion }\end{array}$ \\
\hline
\end{tabular}
Revised version accepted for publication 3 April 1997
Objective comparison of the face in mild and classical de Lange syndrome, through the use of craniofacial pattern profiles, shows marked similarity of patterns at 4 to 9 years; both groups have microbrachycephaly, but the individual dimensions of the mild group are slightly closer to normal than their classical counterparts. The correlation coefficient is high $(0.83)$. In the adult groups, similarity of patterns remains but is less marked. The normalisation of scores in the mild group is more dramatic. The correlation coefficient is lower (0.71).

These objective findings substantiate clinical impressions of a phenotypic dichotomy. Early in life, the craniofacial features in mild de Lange syndrome may be indistinguishable from the classical phenotype and alternative discriminators must be sought in order to identify those subjects in whom the prognosis is more optimistic. Birth weight of more than $2500 \mathrm{~g}$ and absence of major limb anomalies may help in this regard.

$(\mathcal{O}$ Med Genet 1997;34:645-650)

Keywords: de Lange syndrome; classical phenotype; mild phenotype; craniofacial pattern profiles

The facial appearance in classical de Lange syndrome (CdLS) is striking and easily recognisable. The combination of characteristic eyebrows, which are neat, well defined, and arched, as though they had been pencilled in, anteverted nostrils, thin lips, crescent shaped mouth, and long philtrum, whose length may be exaggerated by short nasal height, is quite typical. ${ }^{12}$ In the past, mild CdLS had been discussed mainly in the context of a previously undiagnosed adult, identified after the birth of a classically affected child. Today that phenotypic dichotomy, with classical and mild cases, is well established. Patients with mild CdLS are distinguished by less significant psychomotor retardation. Pre- and postnatal growth deficiency is less marked. Height at 5 years is on the 3rd to 50th centile. ${ }^{3}$ Major malformations are uncommon, although mild limb anomalies may be present. Ironically, behavioural dysfunction may be more evident because of improved developmental skills leading to better mobility and independence. Clearly, diagnosis of mild CdLS is invaluable in order to provide the best information to the family about prognosis, recurrence, and intervention. Birth incidence of de Lange syndrome is approximately 1 in $40000 .^{3}$ The proportion with a mild phenotype is difficult to estimate because features 

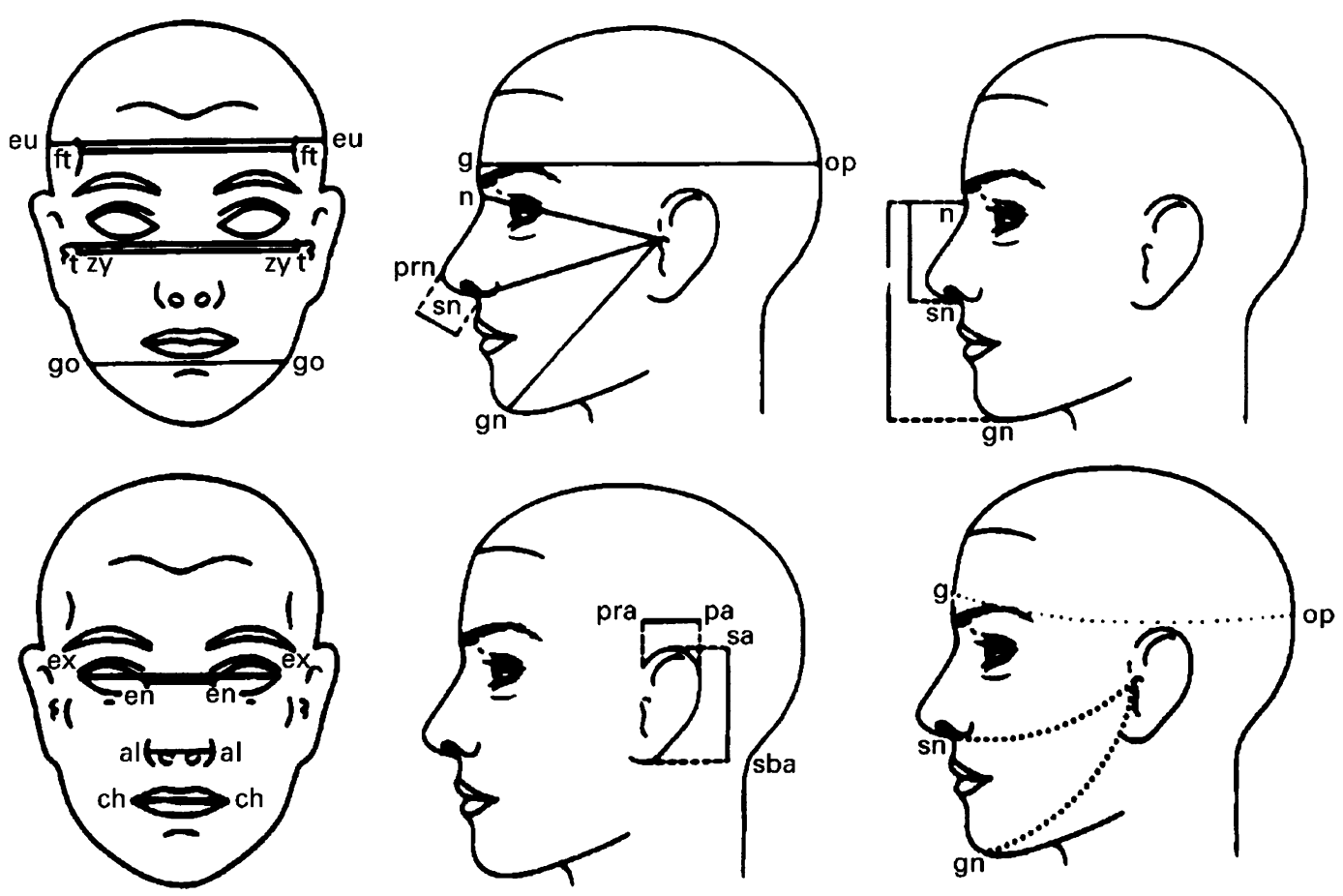

Figure 1 Anthropometric dimensions used in this study. Figure adapted from "Anthropometric facial proportions in medicine", by Farkas LG and Munro IR, Charles C Thomas, Springfield, Illinois, 1987, with permission.

may not be recognisable until 3 years of age; however, in the experience of one of the authors (MI) it is felt to be 20 to $30 \%$ of the total number of subjects with de Lange syndrome.

In this study, the goals were twofold: to evaluate subjects with the mild phenotype both subjectively and anthropometrically to establish whether any age related changes in facial appearance exist, and to determine the basis for discriminating the mild and classical phenotypes and the age at which this would be possible.

Age related changes in facial phenotype are now well recognised ${ }^{4}$ and are increasingly incorporated into syndrome delineation..$^{5-13}$ Anthropometry can enhance subjective or gestalt impressions of facial appearance, particularly if individual measurements are converted into $Z$ scores to produce a pattern profile. ${ }^{14}$ Any dimension characterised by a high or low $\mathrm{Z}$ score is immediately obvious and serves to identify craniofacial areas that are most unusual or characteristic of a particular syndrome. Pattern profiles can be applied across age groups and may show persistence or change in facial appearance with time. They also allow comparison between a single person and a group with a known diagnosis.

To supplement a simple visual appreciation of these pattern profiles, statistical measures of similarity and variability can be calculated. The correlation coefficient, $r_{z}$, is computed from paired $\mathrm{Z}$ scores of two pattern profiles to provide a numerical indication of the similarity of any two patterns. The pattern variability index, $\sigma_{z}$, expresses the degree of dysmorphogenesis as a single number. The more highly patterned a person, the greater the deviation from the reference population and the greater the variability index. ${ }^{15}$
Syndrome specific patterns have been compiled in Wiedemann-Beckwith syndrome, ${ }^{13} 16$ Simpson-Golabi-Behmel syndrome, ${ }^{10}$ hypohidrotic ectodermal dysplasia, ${ }^{17}$ Down syndrome, ${ }^{12}$ and Williams syndrome (Pober, personal communication). These may be used not only for diagnosis of affected subjects but also to assist the identification of carriers of $\mathrm{X}$ linked conditions, such as hypohidrotic ectodermal dysplasia and Simpson-Golabi-Behmel syndrome. ${ }^{10} 17$

\section{Materials and methods}

Forty-five Caucasian subjects with de Lange syndrome, 30 with classical features aged from birth to 21 years and 13 with the mild phenotype aged from 18 months to 30 years, were ascertained. Most were referred to one of the authors (MI). The diagnostic criteria have been published previously. ${ }^{2}$ A series of anthropometric measurements was obtained on each subject (table 1) following the method published by Farkas. ${ }^{18}$ Measurements were recorded to the nearest $0.5 \mathrm{~mm}$ using GPM sliding and spreading, blunt ended calipers, and a paper metric tape measure. These dimensions were chosen to represent craniofacial widths, lengths, depths, and circumferences plus details of ear, eye, nose, and mouth structure (fig 1). For each dimension, age and sex matched normal standards were available. The population norms were derived from measurements of the head and face in 2326 healthy North American Caucasian children and young adults. ${ }^{19}$ Measurements were taken by one of the authors (JEA). The raw data were compared to normal standards and converted to $\mathrm{Z}$ scores to control for age and sex differences. Pattern profiles were compiled for each age and sex. Because of small numbers at certain ages, additional profiles were produced for two 


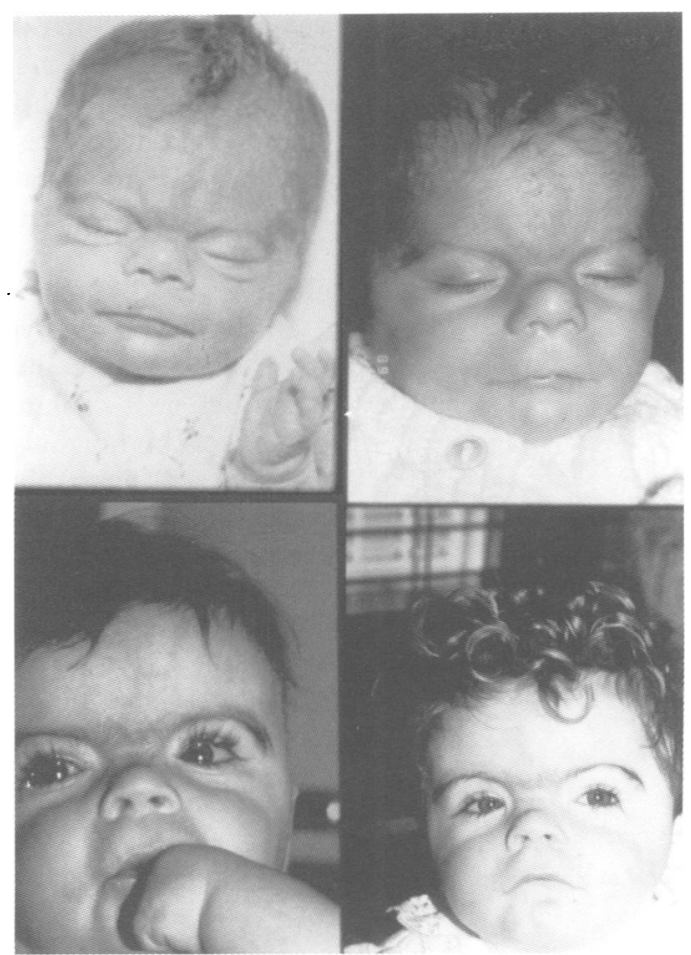

Figure 2 The classical face in infancy.

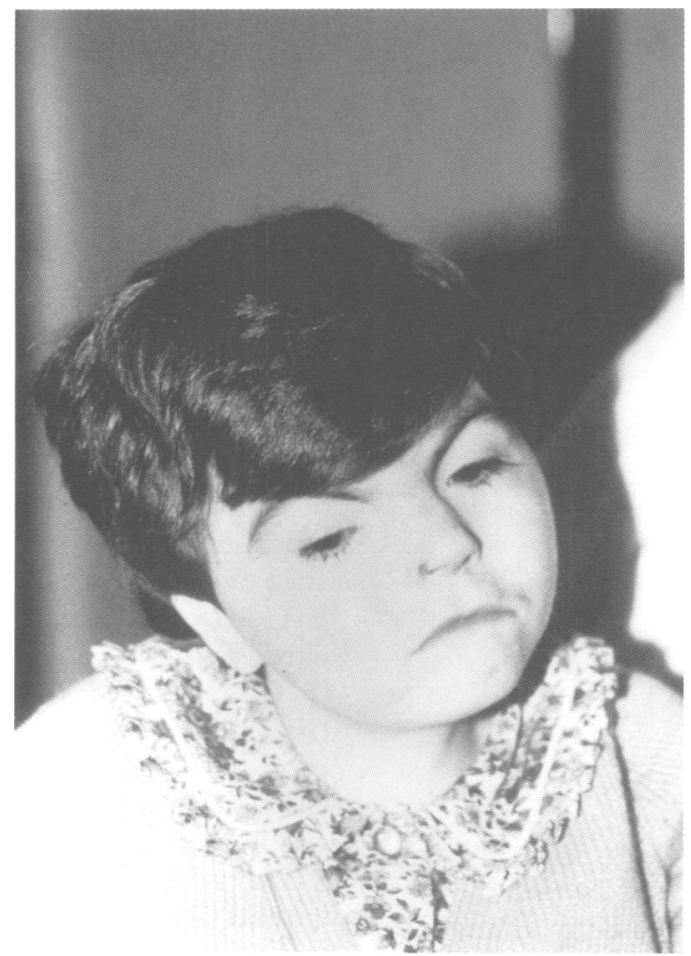

Figure 3 The classical face in adulthood.

groups of subjects, aged 4 to 9 , and 16 and over. Correlation coefficients and variability indices were generated by the Statistical Package for Social Scientists (SPSS), using the methods published by Garn et al..$^{14}$

\section{Results}

The facial appearance of classical CdLS is quite distinctive. Despite past emphasis on bushy eyebrows, synophrys, and hypertrichosis, Ireland et $a l^{12}$ have made a compelling argument for diagnostic weight to be given to the combination of characteristic eyebrows,

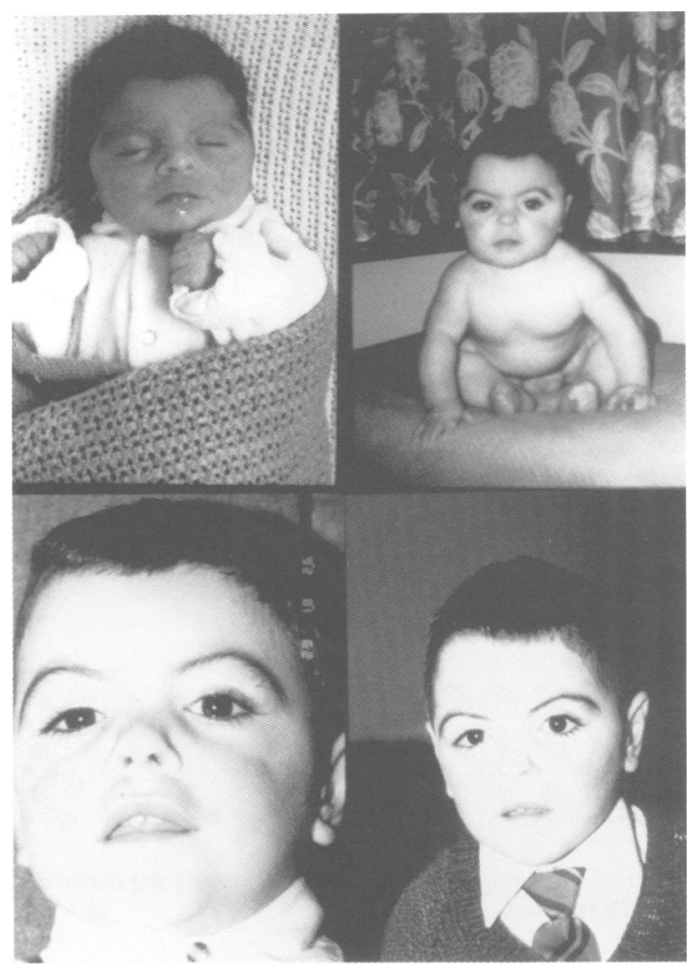

Figure 4 Mild de Lange syndrome: the face of the infant and young child.

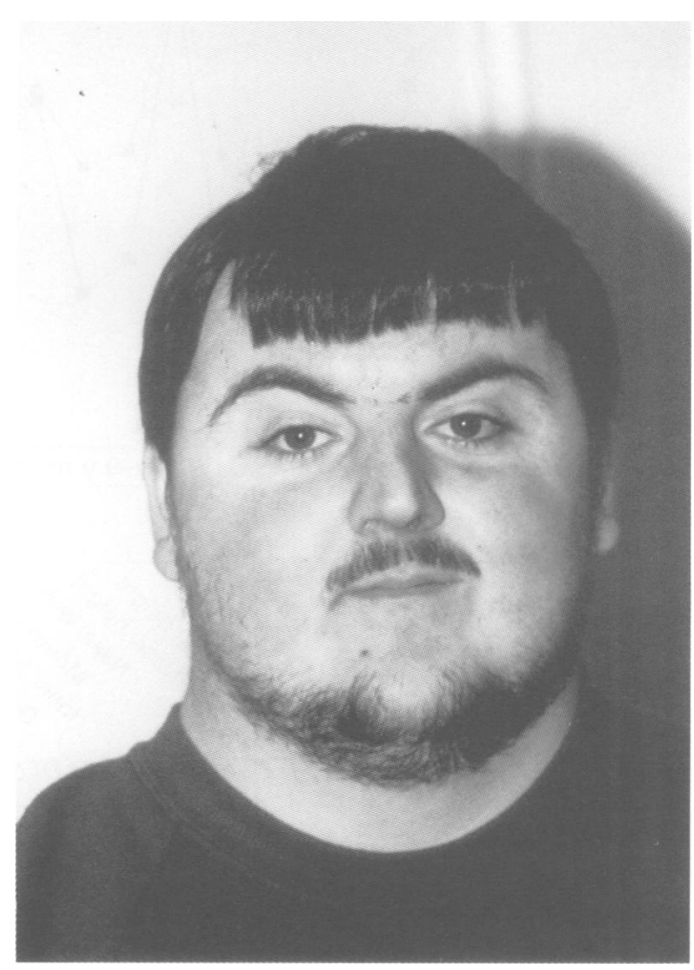

Figure 5 The adult with mild CdLS.

which are neat, well defined, and arched, as though they had been pencilled in, anteverted nostrils, thin lips, crescent shaped mouth, and long philtrum, whose length may be exaggerated by short nasal height.

In the newborn the eyebrows may be poorly defined; however, the other features (anteverted nares, lips, mouth shape, and long philtrum) are quite recognisable. There is microbrachycephaly and a short neck (fig 2). By infancy, classical eyebrow shape is obvious and the other features persist. The lashes are thick and curled. In childhood, as part of the 


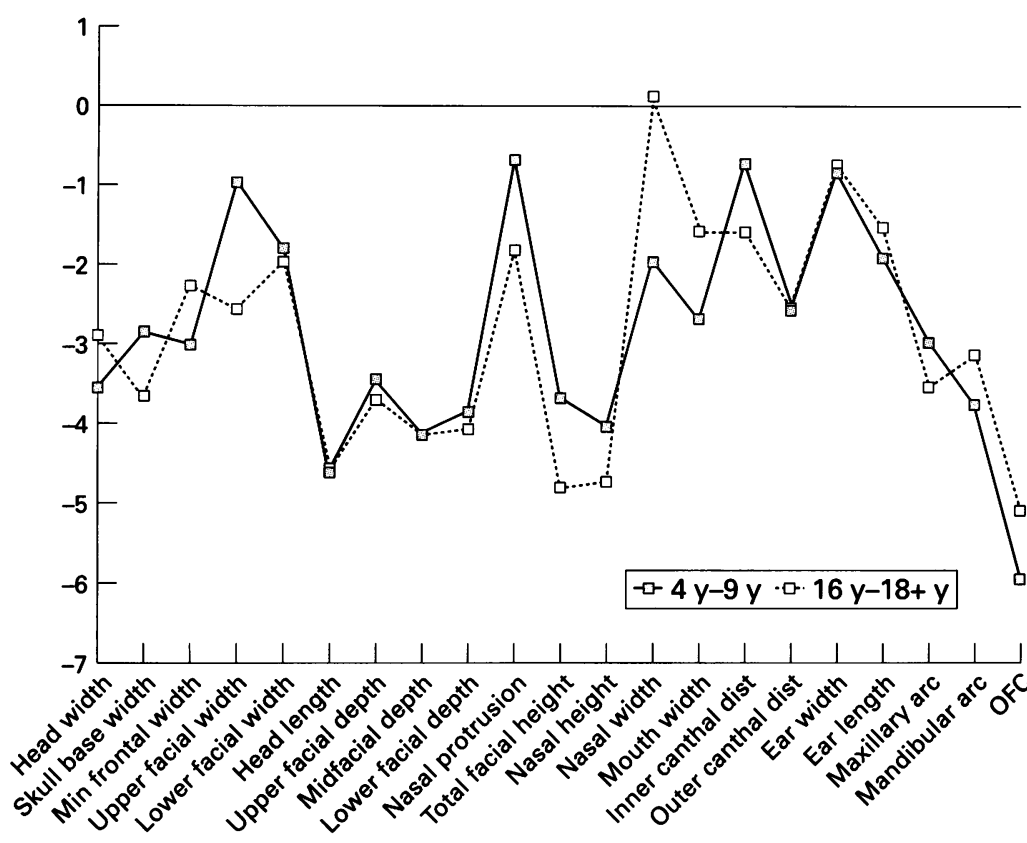

Figure 6 Craniofacial pattern profile comparing classical DLS at 4 to 9 years and in the over 16 year old.

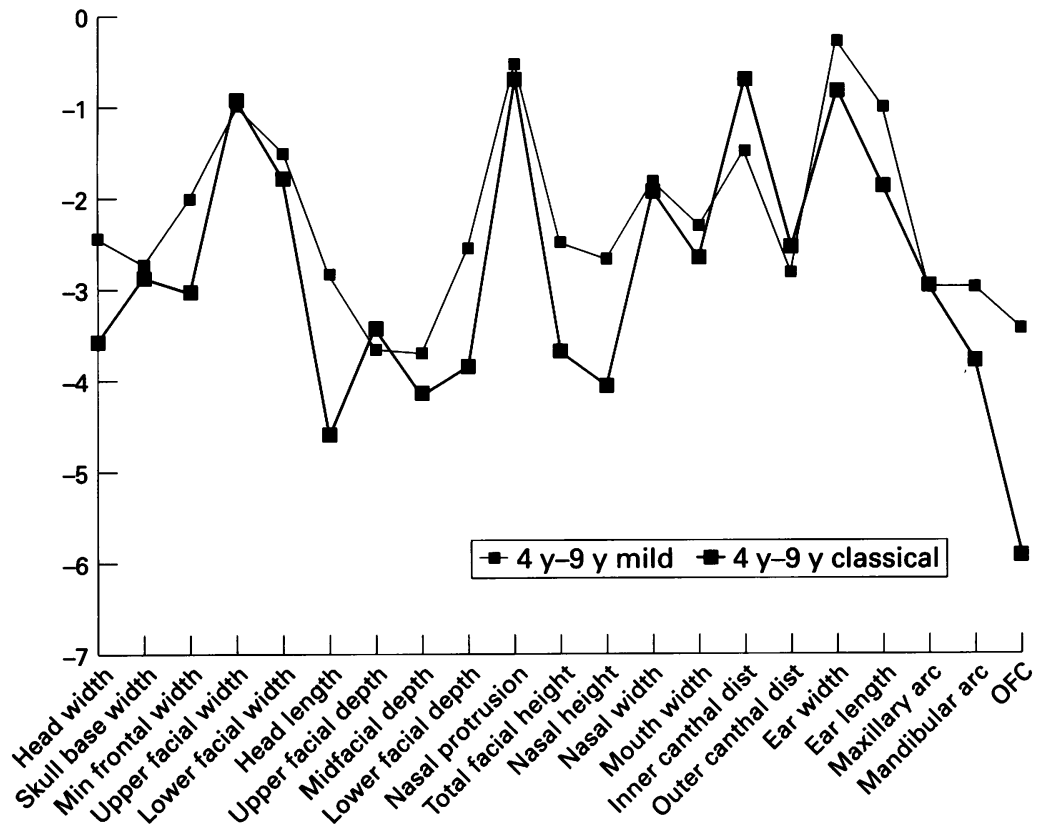

Figure 7 Pattern profile comparing classical and mild DLS in the 4 to 9 year group.

normal process of facial growth, the face lengthens. The nasal root and tip become more prominent, which will eventually change the orientation of the nares to downward facing. Nasal height increases, which may diminish the impression of a long philtrum. Lip and mouth shape are unchanged. In the adolescent, eyebrows become bushier, less "pencilled" but still well defined. By adulthood, the face is more oblong, as we would expect given the increase in vertical dimensions, and the nose appears longer and more prominent. The jaw is slightly squared. The other typical features persist (fig 3).

In the evaluation of the mild phenotype, the early situation is a little more complex. Some neonates with mild CdLS will manifest classical features at birth, while in others the features do not become obvious until the age of 2 to 3 years. The newborn displays those typical eyebrows, anteverted nares, long philtrum, and mouth shape. The lips are a little fuller. Even though the characteristic features are present in the newborn and infant, the overall impression is perhaps less striking. In part, this may be a function of improved facial expression and alertness (fig 4).

In childhood, the typical appearance is much more recognisable, but in the adult the features are much less striking (fig 5). The philtrum appears to be normal in length, partly perhaps because of near normal nasal height and protrusion, and partly because the upper vermilion is fuller and everted. Despite fullness of the lips, the crescent shaped mouth is evident. The eyebrows extend laterally and are full and bushy. The nose is well developed and finely shaped, but still mildly short.

In order to obtain a data set of adequate size, the pattern profile analysis will concentrate on the two largest subgroups, with about equal representation of mild and classical cases. The two groups are 4 to 9 years, in which there are seven classical and eight mild cases, and age 16 and above, in which there are five classical and four mild cases. Fortuitously, they span the child to adult transition.

The first profile compares classical CdLS in childhood and adulthood (fig 6). There is clear concordance of patterns. This subjective impression is substantiated by a correlation coefficient of 0.78 . This profile similarity does not imply an absence of change over time, but suggests that normal ageing occurs, superimposed on the characteristic pattern associated with the syndrome. That characteristic pattern has several components: OFC is markedly reduced (-6 SD), with cranial length less than width (brachycephaly); facial widths are closer to normal than depths and heights; nasal height is reduced, which may contribute to the appearance of a long philtrum.

The second profile evaluates the 4 to 9 year olds, comparing mild and classical patterns (fig 7). Concordance is striking and the correlation coefficient is even greater at 0.83 . Microbrachycephaly is present in both profiles, but the individual dimensions in mild CdLS (OFC, head width, and length) are all closer to normal.

The subjective change in age related gestalt in mild CdLS is confirmed in the third profile, comparing children and adults with the mild phenotype (fig 8). There is less concordance between the age groups. Correlation coefficient is 0.76 . The overall pattern is quite similar, particularly in craniofacial widths, lengths, depths, heights, and circumferences. However, the adult dimensions are obviously significantly closer to normal. OFC is almost normal, although brachycephaly persists, and lower facial width is increased.

The final profile comparing adults with classical and mild CdLS reinforces the normalisation of dimensions in adults with mild CdLS (fig 9). Some pattern concordance remains, with correlation coefficient of 0.71 ; however, this is the weakest correlation of all. 


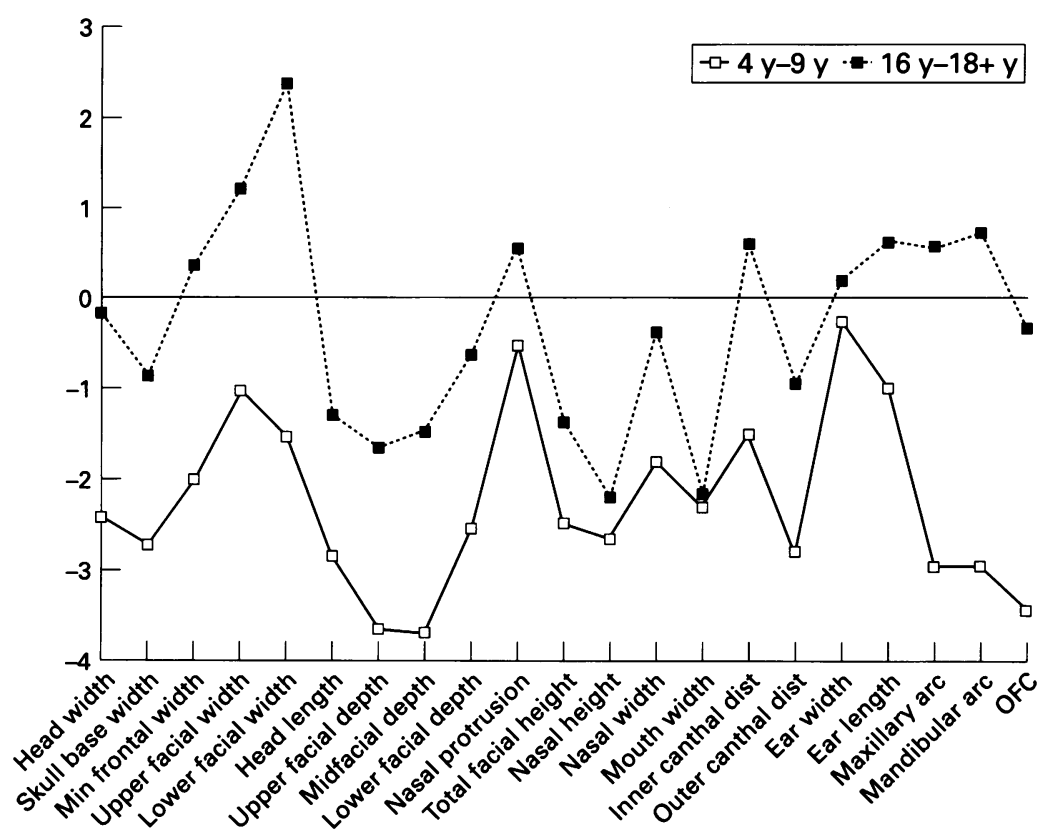

Figure 8 Pattern profile of mild DLS in childhood and adulthood.

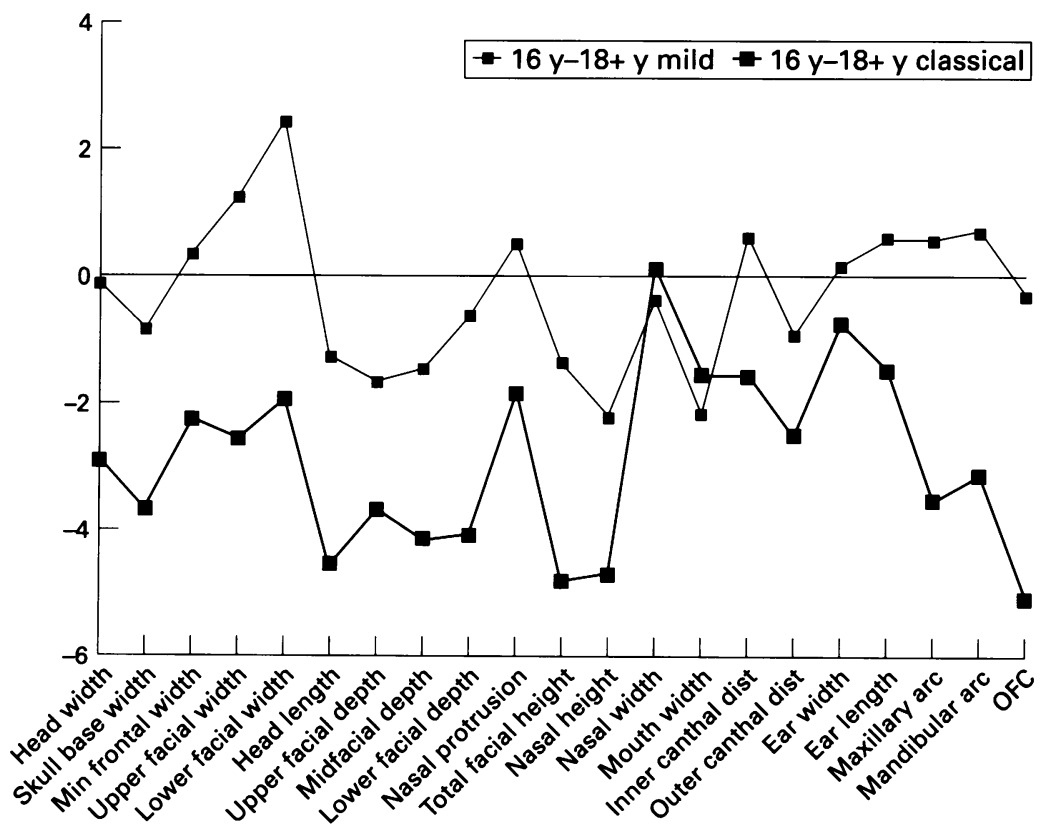

Figure 9 Pattern profile comparing adults with classical and mild DLS.

\section{Discussion}

These objective findings substantiate clinical impressions of a phenotypic dichotomy. Early in life, the craniofacial features in mild CdLS may be indistinguishable from the classical phenotype and alternative discriminators must be sought in order to identify those subjects in whom the prognosis is more optimistic. Saal et $a l^{20}$ suggest that birth weight greater than 2500 $\mathrm{g}$ and absence of major limb anomalies may help in this regard. Ireland ${ }^{3}$ has summarised her experience of over 100 affected subjects. Birth weight in classical de Lange syndrome is less than 3\%, while in mild de Lange syndrome it is 3 to $10 \%$. Height at 5 years is generally in the normal range in mild de Lange syndrome (3 to $50 \%$ ). Severe mental retardation in the classical phenotype contrasts with borderline to mild deficiency in the mildly affected subjects. Normal intelligence has not been recorded in mild de Lange syndrome. Small hands are present in both groups, but major limb anomalies are not seen in mild de Lange syndrome. Heart defects are less common in the mild phenotype ( $2 \%$ rather than $18 \%$ ). Two groups (Steve Braddock, San Diego and Merlin Butler, Nashville) suggest that there might be a characteristic MCPP in mild CdLS. It is important to remember that, in some cases, the mild gestalt may not become recognisable until around the age of 3 years.

Most cases of de Lange syndrome are sporadic and are thought to result from a new dominant mutation. More mildly affected subjects, who live independently, have had children, both affected and unaffected, in keeping with this dominant hypothesis. ${ }^{21}$ It is more common to see females transmitting the trait. So far, all affected offspring have been mildly affected. Adults with mildly suggestive features deserve detailed review of early photographs to define those with an increased risk for an affected child. A few occurrences in a sibship have been described and probably result from germinal mosaicism. ${ }^{22}$

The pathogenetic mechanisms underlying the dichotomy remain to be elucidated but several hypotheses are tenable. Allele specific phenotypes are one possibility. There may be two de Lange syndrome genes in the same developmental pathway, with severity dependent on the point at which the pathway is disrupted. Mild and classical de Lange syndromes may result from the same mutation with other genes modifying prognosis. Lastly, it is possible that mild de Lange syndrome is a phenocopy, although there is no evidence to support this, and it seems the least likely explanation.

We are extremely grateful to Dr John Burn, who facilitated patient ascertainment, the many referring physicians in the UK and Canada, and the Cornelia de Lange syndrome UK executive and members without whose cooperation and support this study would not have been possible. The study was partly this study would not have been possible. The study was partly
supported by a grant from the Children's Hospital of Eastern supported by a grant from the Children's Hospital of Eastern Ontario Research Foun
Council (UK) (MI)

1 Ireland M, Burn J. Cornelia de Lange syndrome - photo essay. Clin Dysmorphol 1993;2:151-60.

2 Ireland $M$, Donnai $D$, Burn J. Brachmann-de Lange syndrome. Delineation of the clinical phenotype. Am $\mathcal{F ~ M e d ~}$ Genet 1993;47:959-64.

3 Ireland M. Cornelia de Lange syndrome: clinical features, common complications and long-term prognosis. Curr Pediatr 1996;6:69-73.

4 Allanson JE. Time and natural history: The changing face. $\mathcal{J}$ Craniofac Genet Dev Biol 1989;9:21-8.

5 Allanson JE, Hall JG, Hughes HE, Preus M, Witt D. Noonan syndrome: an evolving phenotype. Am $\mathcal{f} \mathrm{Med}$ Genet 1985;21:507-14.

6 Allanson JE. G syndrome: an unusual family. $\mathrm{Am} f \mathrm{Med}$ Genet 1988;31:637-42.

7 Allanson JE. Rubinstein-Taybi syndrome: the changing face. Am f Med Genet Suppl 1990;6:38-41.

8 Porteous MEM, Goudie DR. Aarskog syndrome. $f \mathrm{Med}$ Genet 1991;28:44-7.

9 Lopez-Rangel E, Maurice M, McGillivray B, Friedman JM. Williams syndrome in adults. Am $\mathcal{F}$ Med Genet 1992;44: 720-9.

10 Hughes-Benzie RM, Hunter AGW, Allanson JE, Mackenzie A. Detailed study of an extended family with SimpsonGolabi-Behmel syndrome. Proc Greenwood Genet Center 1992;11:109.

11 Fryns JP. Aarskog syndrome: the changing phenotype with age. Am $\mathcal{F}$ Med Genet 1992;43:420-7.

12 Allanson JE, O'Hara P, Farkas LF, Nair R. Anthropometric craniofacial pattern profiles in Down syndrome. Am $\mathcal{F}$ Med craniofacial pattern pro

13 Hunter AGW, Allanson JE. A follow up study of patients with Wiedemann-Beckwith syndrome with emphasis on 
the change in facial appearance over time. Am $\mathfrak{f}$ Med Genet 1994;51:102-7.

14 Garn SM, Smith BH, Lavelle M. Applications of pattern profile analysis to malformations of the head and face. Radiology 1984;150:683-90.

15 Garn SM, Smith BH, Lavelle M. Quantification of dysmorphogenesis: pattern variability index, $\theta_{2} . A m f$ Radiol 1985;144:365-9.

16 Ward RE, Escobar LF, Carlin ME, Haines JL. Quantitative analysis of the face in the Beckwith Wiedemann syndrom and detection of minimally affected gene carriers. $A m$ Hum Genet 1990;47:82A.

17 Ward RE, Bixler D. Anthropometric analysis of the face in hypohidrotic ectodermal dysplasia: a family study. $A m \mathcal{F}$ Phys Anthropol 1987;74:453-8.
18 Farkas LG. Anthropometry of the head and face in medicine. New York: Elsevier, 1981.

19 Farkas LG. Anthropometry of the head and face in medicine. 2nd ed. New York: Raven Press, 1994.

20 Saal HM, Samango-Sprouse CA, Rodnan LA, Rosenbaum $\mathrm{KN}$, Custer DA. Brachmann-de Lange syndrome with normal IQ. Am 7 Med Genet 1993;47:995-8.

21 Chodirker BN, Chudley AE. Male-to-male transmission of mild Brachmann-de Lange syndrome. Am 7 Med Genet 1994;52:331-3.

22 Fryns JP, Dereymaeker AM, Hoefnagels M, D'Hondt F, Mertens G, Van den Berghe $H$. The Brachmann-de Lange syndrome in two siblings of normal parents. Clin Genet 1987;31:413-15.

$\frac{c}{\mathrm{D}}$

ه

$\stackrel{\mathbb{\mathbb { Q }}}{\rightarrow}$

$\stackrel{\vec{F}}{\stackrel{9}{+}}$

음

$\frac{\bar{\omega}}{\frac{1}{D}}$ 